\title{
Can PD-L1 tumor proportion score be used as the key to unlocking the KEYNOTE studies of pembrolizumab in advanced lung cancer?
}

\author{
Andrew J. Piper", Kartik Sehgal", Daniel B. Costa, Deepa Rangachari \\ Department of Medicine, Division of Medical Oncology, Beth Israel Deaconess Medical Center (a member of Beth Israel Lahey Health), Harvard \\ Medical School, Boston, MA, USA \\ \#These authors contributed equally to this work. \\ Correspondence to: Deepa Rangachari, MD. Division of Medical Oncology, Beth Israel Deaconess Medical Center, 330 Brookline Avenue, Boston, MA \\ 02215, USA. Email: drangach@bidmc.harvard.edu. \\ Provenance: This is an invited article commissioned by the Section Editor Kaiping Zhang, PhD (AME College, AME Group, China). \\ Comment on: Reck M, Rodríguez-Abreu D, Robinson AG, et al. Updated analysis of KEYNOTE-024: pembrolizumab versus platinum-based \\ chemotherapy for Advanced non-small-cell lung cancer with PD-L1 tumor proportion score of 50\% or greater. J Clin Oncol 2019;37:537-46.
}

Submitted May 14, 2019. Accepted for publication May 20, 2019.

doi: $10.21037 /$ tlcr.2019.05.12

View this article at: http://dx.doi.org/10.21037/tlcr.2019.05.12

\section{Introduction}

Despite substantial advancements in the management of advanced non-small cell lung cancer (NSCLC) made possible with application of molecularly targeted and immune-based strategies over the last two decades, significant challenges remain (1). On the basis of these advancements, the evidence-based initial management of advanced/metastatic NSCLC is now necessarily defined by tumor molecular and immune biomarkers so as to permit optimal pairing of patients with the most efficacious and least toxic therapies-and with previously unseen extension of survival in multiple large studies in these selected populations (2-5). Reflecting this evolving reality, evidencebased international guidelines currently recommend tumor testing for epidermal growth factor receptor (EGFR), anaplastic lymphoma kinase (ALK), ROS proto-oncogene 1 (ROS1), and $B$-raf proto-oncogene $(B R A F)$ mutations/gene rearrangements as well as programmed death ligand 1 (PD-L1) tumor proportion score (TPS) as part of the mandatory upfront molecular profiling paradigm for all treatment-eligible patients presenting with advanced/metastatic non-squamous NSCLC-regardless of clinical or demographic factors (i.e., tobacco history) $(6,7)$. In squamous tumors, where the advances of targeted molecular therapies have remained disappointing to date, minimum requirements for testing include tumor PD-L1 TPS, with consideration of additional genomic testing based on clinical factors.

However, the vast majority of patients with advanced NSCLC will not have an actionable driver oncogene alteration amenable to use of a precision tyrosine kinase inhibitor (TKI), and it is in this substantial cohort of patients where advances in immune-based therapies have radically transformed the therapeutic landscape $(1,8)$. Currently, three different immune checkpoint inhibitors (ICIs) nivolumab (anti-PD1), pembrolizumab (anti-PD1), and atezolizumab (anti-PD-L1) have secured approval from the United States Food and Drug Administration (FDA) for use in patients with advanced stage NSCLC. These ICIs alone and in combination with platinum doublet chemotherapy have demonstrated superior and durable overall survival (OS) with favorable toxicity profiles and quality of life measures when compared with chemotherapy-in both upfront and subsequent lines of treatment (9-13). These advancements have led to an implosion of the long-held hegemony of platinum doublet chemotherapy for the initial management of advanced NSCLC without actionable genomic alterations (i.e., in $E G F R, A L K$, and others), where the evidence-based standard of care is now necessarily ICI \pm chemotherapy. On the basis of progress made in several landmark studies to date, pembrolizumab is currently FDA approved in the following settings for advanced NSCLC: 
(I) first-line monotherapy in tumors without EGFR/ALK alterations and PD-L1 TPS $\geq 1 \%$; (II) first-line combination therapy with platinum doublet chemotherapy in tumors without EGFR/ALK alterations, irrespective of tumor PDL1 TPS; and (III) following failure of platinum doublet or targeted therapies in ICI-naïve patients with tumor PD-L1 TPS $\geq 1 \%$.

\section{From last to first: moving pembrolizumab into the starting lineup}

KEYNOTE-024 was a landmark phase III trial which randomized 305 previously untreated patients with advanced NSCLC and no actionable alterations in EGFR/ALK to receive either platinum doublet chemotherapy or single agent pembrolizumab (14). As with all of the KEYNOTE studies, tumor PD-L1 status was determined using the $22 \mathrm{C} 3$ pharmDx immunohistochemistry companion diagnostic assay. The study achieved its primary endpoint of improved progression-free survival (PFS) as well as the secondary endpoint of improved OS. Recently published updated OS data with extended follow-up demonstrated a median OS of $30 v s .14 .2$ months with pembrolizumab vs. platinum doublet chemotherapy, respectively (9). Notably, 82 patients in the chemotherapy arm crossed over to pembrolizumab. However, even with statistical analysis to adjust for the crossover effect, upfront use of pembrolizumab maintained its survival advantage, thus demonstrating the durable superiority of pembrolizumab over chemotherapy earlier in the disease course in this biomarker-driven subset of patients. Moreover, treatmentrelated serious adverse events (AEs) [defined as $\geq$ grade 3 using common terminology criteria for adverse events (CTCAE)] were seen less frequently with pembrolizumab vs. chemotherapy: $31.2 \%$ vs. $53.3 \%$, respectively.

In the ensuing KEYNOTE-042 trial, patients with any degree of tumor PD-L1 positivity (i.e., PD-L1 TPS $\geq 1 \%$ ) were randomized to receive frontline pembrolizumab monotherapy $v s$. platinum doublet chemotherapy. Here again, the OS in the overall study group was superior in the pembrolizumab arm (15). In predefined subgroups, an increasing degree of tumor PD-L1 positivity was associated with an increasing magnitude of survival benefit for pembrolizumab vs. chemotherapy: (I) for PD-L1 TPS $\geq 1 \%$, median OS 16.7 vs. 12.1 months [hazard ratio (HR) 0.81 , respectively; (II) for PD-L1 TPS $\geq 20 \%$, median OS 17.7 vs. 13 months (HR 0.77), respectively; (II) for PDL1 TPS $\geq 50 \%$, median OS 20 vs. 12.2 months (HR 0.69), respectively. As in other studies, treatment-related serious AEs were again less commonly seen with pembrolizumab $(18 \%)$ than with chemotherapy $(41 \%)$. Notably, a prespecified exploratory analysis of those patients with tumors harboring PD-L1 TPS 1-49\% showed no significant improvement in OS with pembrolizumab as compared to chemotherapy (median OS of 13.4 vs. 12.1 months, HR 0.92, respectively). Thus, much of the survival advantage seen appears to have been driven by the same population of patients previously identified in KEYNOTE-024-i.e., those with tumor PD-L1 TPS $\geq 50 \%$.

Yet even despite these advances, a substantial proportion of patients with advanced NSCLC in need of effective palliation of their disease will not stand to benefit from single agent ICI or targeted therapy alone. Extension of benefit to this subset of patients has necessitated exploration of combination therapy strategies. In KEYNOTE-189 (non-squamous) and KEYNOTE-407 (squamous), pembrolizumab in combination with platinum doublet chemotherapy $v s$. platinum doublet chemotherapy alone established the critical importance of ICI in the upfront treatment strategy of these patients. Both studies, which enrolled patients with tumors lacking actionable alterations in EGFR/ALK and with any tumor PD-L1 TPS, met their primary endpoints of improved OS and PFS with chemoimmunotherapy $v s$. chemotherapy alone $(10,16)$. Here again, while a higher degree of tumor PD-L1 expression was associated with greater median PFS and OS, all groups receiving combination chemoimmunotherapy performed better than those receiving chemotherapy alone. Specifically, in patients with tumor PD-L1 TPS $<1 \%$, the HRs for death with chemoimmunotherapy $v s$. chemotherapy alone were 0.59 and 0.61 in patients with non-squamous and squamous tumors, respectively. However, increasing treatment efficacy did come at the cost of increased rates of or life-threatening AEs associated with chemoimmunotherapy: $67.2 \%$ in KEYNOTE-189 and $69.8 \%$ in KEYNOTE-407.

\section{Pearls and pitfalls of patient and biomarker selection}

Despite these encouraging results, many important questions still remain regarding the optimal use of ICIs and applicability of clinical trial results in the real-world setting. As is often the case, these trials excluded patients and circumstances commonly encountered in day-to-day clinical practice, i.e., patients with Eastern Cooperative 
Oncology Group (ECOG) performance status (PS) $\geq 2$, antecedent autoimmune disease, chronic viral infections such as human immunodeficiency virus (HIV) and hepatitis $\mathrm{B} / \mathrm{C}$, and brain metastases. Given the potential for benefit and/or harm associated with use of ICIs in these subpopulations, much effort has been made in the postapproval period to explore the limited evidence available to guide use of ICIs in these individuals $(17,18)$. Systematic reviews and case series in advanced cancer patients have suggested that ICIs are effective and might be safely administered in patients with HIV infection $(19,20)$, preexisting autoimmune disease (21), and small/asymptomatic untreated brain metastases (22).

The initial promise of tumor PD-L1 TPS as a distinctive biomarker notwithstanding, optimal biomarker selection for treatment with ICIs remains an area of continued evolution. As has become increasingly evident, tumor PD-L1 alone is unlikely to maximally identify all patients likely to derive benefit from ICIs-and even those with high tumor PDL1 do not uniformly achieve the desired brisk and durable benefit. Notably, the comparator arm in both frontline studies of single agent pembrolizumab was the now defunct historical control of platinum doublet chemotherapy, rather than combination chemoimmunotherapy; as such, a persistent area of uncertainty remains regarding when and in whom chemotherapy must necessarily be added to the immunotherapy backbone-particularly in those patients with tumor PD-L1 TPS of $\geq 1 \%$, where pembrolizumab alone is now a viable option. Further, PD-L1 alone is unlikely to capture the full scope of potential benefit with ICI use, and evolution of multiplex prediction tools that incorporate assessment of other independent biomarkers including tumor mutation burden (TMB), tumor infiltrating lymphocytes, microsatellite instability, mutations in DNA damage repair pathways, tobacco exposure, and presence/ absence of tumor suppressor genes that affect innate immune function (amongst others) are likely to become both necessary and feasible (23).

Exploration of a tumor's co-mutation profile is also likely to affect ICI efficacy and durability. Use of ICI monotherapy in patients with tumors harboring actionable alterations in $E G F R / A L K$ has been consistently associated with disappointing results (24-26). Further, in a recent analysis, presence of targetable driver mutations, serine/ threonine kinase 11 (STK11) mutation, absence of tumor PD-L1 expression, and low TMB were identified as predictors of inferior outcomes associated with use of ICI monotherapy in this setting (27). Perhaps of even greater concern, there is mounting evidence that use of ICIs sequentially or in combination with TKIs like crizotinib in $A L K-$, ROS-1-, and MET-altered NSCLC as well as osimertinib in EGFR-mutated NSCLC can be associated with high-grade TKI- and ICI-related AEs, in some cases requiring permanent discontinuation of what might have otherwise been a highly efficacious therapy $(28,29)$. These factors all underscore the importance of upfront, high fidelity, high efficiency genotyping of advanced NSCLC at initial diagnosis and prior to starting palliative systemic therapy—not only to optimize clinical efficacy, but also to avoid unnecessary toxicity.

\section{Our approach to first-line therapy: optimizing therapeutic stratification}

The current evidence-based standard of care for upfront management of advanced NSCLC now necessarily relies on therapeutic stratification on the basis of tumor histology, genomic biomarkers, and PD-L1 immunohistochemistry (Figure 1). In all patients whose tumors lack actionable driver oncogene alterations and who have no contraindications to immunotherapeutic agents, pembrolizumab with/without platinum doublet chemotherapy is the standard. Although the combination of atezolizumab and bevacizumab in conjunction with platinum doublet chemotherapy has also been recently approved for management of previously untreated non-squamous NSCLC, we do not routinely utilize this regimen in the clinic due to augmented toxicity with modest incremental benefit. For patients whose tumors do not express PD-L1 (i.e., TPS $<1 \%$ ), pembrolizumab in combination with platinum doublet chemotherapy is the universally recommended approach.

Thus, the major challenge lies in optimal selection between pembrolizumab monotherapy or combined with platinum doublet chemotherapy for the considerable number of patients with PD-L1 TPS 1-49\%. Though the toxicity profile of single agent pembrolizumab is superior to that of chemoimmunotherapy and despite recent FDA approval for this indication, we continue to favor pembrolizumab in combination with platinum doublet chemotherapy for those patients with adequate PS and end organ function; if there are concerns regarding comorbidities and/or toxicity with otherwise adequate PS, then single agent pembrolizumab is a viable alternative. This preference is on the basis of lack of proven OS benefit in the exploratory analysis of the TPS $1-49 \%$ subgroup in KEYNOTE-042 and especially given the 


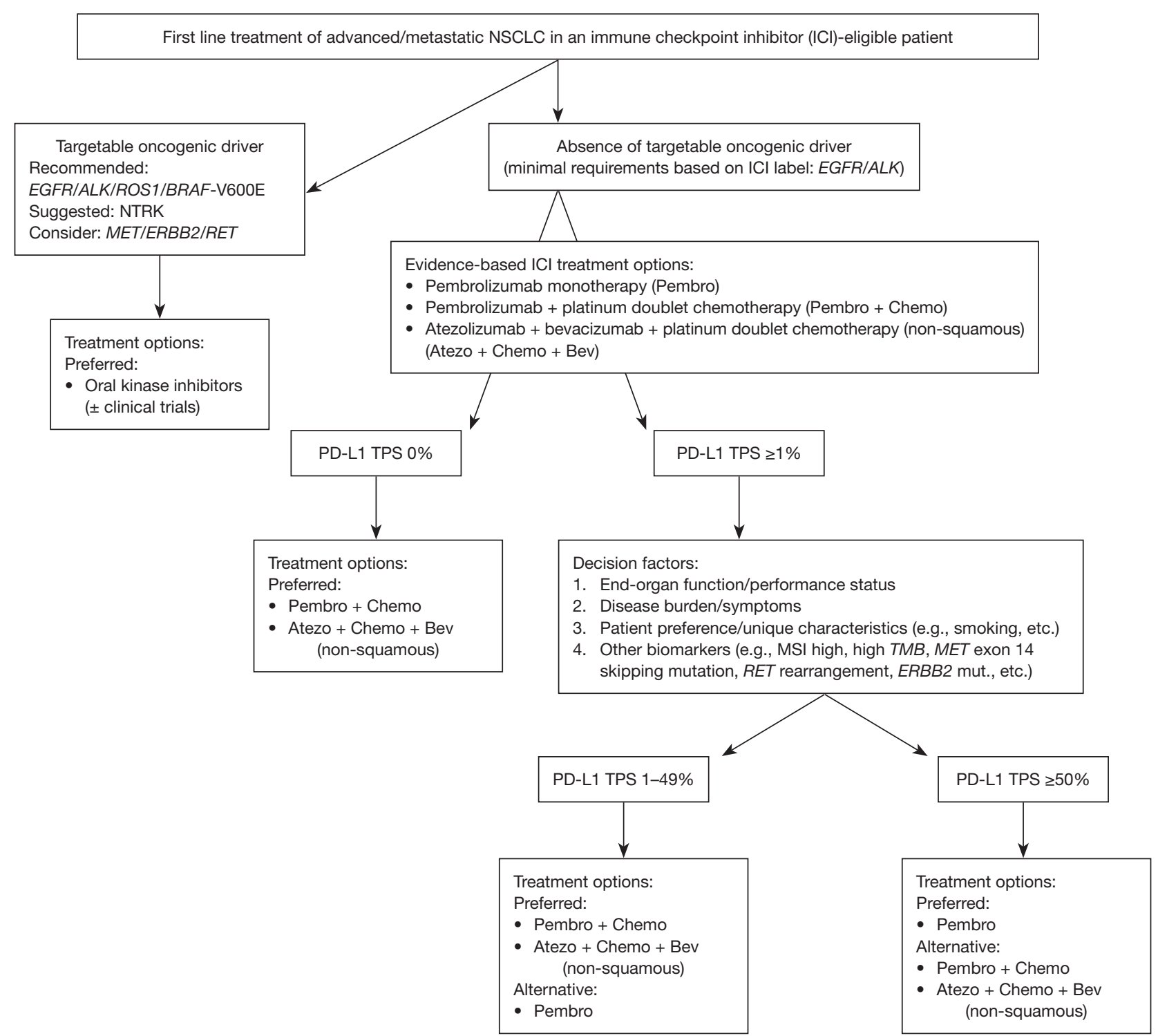

Figure 1 Approach to selection of first line therapy options in advanced/metastatic NSCLC. NSCLC, non-small cell lung cancer; TMB, tumor mutational burden. mut. = mutation.

well-established PFS and OS benefit with combination chemoimmunotherapy in this cohort in KEYNOTE-189 and KEYNOTE-407 $(10,15,16)$.

For those patients with PD-L1 TPS $\geq 50 \%$ and without actionable genomic alterations, the optimal selection between pembrolizumab alone $v s$. in combination with chemotherapy is perhaps similarly uncertain, especially given the sustained survival advantage associated with pembrolizumab use that has persisted even despite significant and robust analysis of the crossover effects $(9,14)$. Within the limitations of cross-trial comparisons between the four frontline KEYNOTE studies, pembrolizumab when combined with chemotherapy led to higher ORR and lower HR for death in all subgroups as compared to those seen with pembrolizumab monotherapy (Table 1). However, not unexpectedly, the rates of serious AEs were higher with the chemoimmunotherapy combination as compared to pembrolizumab alone. Thus, for most patients with tumors having PD-L1 TPS $\geq 50 \%$ and without actionable oncogenic alterations, single agent pembrolizumab is our preferred regimen - with the caveat of treatment-eligible patients with rapidly progressive or bulky symptomatic 
Table 1 Summary of the phase III KEYNOTE studies in advanced NSCLC using PD-L1 TPS

\begin{tabular}{|c|c|c|c|c|}
\hline \multirow{2}{*}{ PD-L1 TPS } & \multicolumn{2}{|c|}{ Pembrolizumab (P) vs. Chemotherapy (C) } & \multicolumn{2}{|c|}{$\begin{array}{l}\text { Pembrolizumab + Chemotherapy (PC) vs. } \\
\text { Chemotherapy (C) }\end{array}$} \\
\hline & KEYNOTE-024 & KEYNOTE-042 & $\begin{array}{l}\text { KEYNOTE-189 } \\
\text { (non-squamous) }\end{array}$ & $\begin{array}{l}\text { KEYNOTE-407 } \\
\text { (squamous) }\end{array}$ \\
\hline \multicolumn{5}{|l|}{$\geq 50 \%$} \\
\hline Median OS (months) & $30(\mathrm{P})$ vs. $14.2(\mathrm{C})^{*}$ & $20(\mathrm{P})$ vs. $12.2(\mathrm{C})$ & - & NR (PC) vs. NR (C) \\
\hline Median PFS (months) & $10.3(\mathrm{P})$ vs. $6.0(\mathrm{C})$ & $7.1(\mathrm{P})$ vs. $6.4(\mathrm{C})$ & - & $8.0(\mathrm{PC})$ vs. $4.2(\mathrm{C})$ \\
\hline HR $(95 \% \mathrm{Cl})$ & $0.50(0.37-0.68)$ & $0.81(0.67-0.99)$ & $0.36(0.25-0.52)$ & $0.37(0.24-0.58)$ \\
\hline ORR (\%) & $44.8(\mathrm{P})$ vs. $27.8(\mathrm{C})$ & $39.5(\mathrm{P})$ vs. $32.0(\mathrm{C})$ & $61.4(\mathrm{PC})$ vs. $22.9(\mathrm{C})$ & $60.3(\mathrm{PC})$ vs. $32.9(\mathrm{C})$ \\
\hline \multicolumn{5}{|l|}{$1-49 \%$} \\
\hline Median PFS (months) & - & - & - & $7.2(\mathrm{PC})$ vs. $5.2(\mathrm{C})$ \\
\hline HR (95\% Cl) & - & - & $0.55(0.37-0.81)$ & $0.56(0.39-0.80)$ \\
\hline ORR (\%) & - & - & 48.4 (P) vs. $20.7(\mathrm{C})$ & 49.5 (PC) vs. $41.3(\mathrm{C})$ \\
\hline \multicolumn{5}{|l|}{$\geq 1 \%$} \\
\hline Median OS (months) & - & $16.7(\mathrm{P})$ vs. $12.1(\mathrm{C})$ & - & - \\
\hline $\mathrm{HR}(95 \% \mathrm{Cl})$ & - & $0.81(0.71-0.93)$ & $0.47(0.34-0.66)$ & $0.65(0.45-0.92)$ \\
\hline Median PFS (months) & - & $5.4(\mathrm{P})$ vs. $6.5(\mathrm{C})$ & - & - \\
\hline HR (95\% Cl) & - & - & $0.59(0.38-0.92)$ & $0.61(0.38-0.98)$ \\
\hline Median PFS (months) & - & - & - & $6.3(\mathrm{PC})$ vs. $5.3(\mathrm{C})$ \\
\hline $\mathrm{HR}(95 \% \mathrm{Cl})$ & - & - & $0.75(0.53-1.05)$ & $0.68(0.47-0.98)$ \\
\hline ORR (\%) & - & - & $32.3(\mathrm{P})$ vs. $14.3(\mathrm{C})$ & 63.2 (PC) vs. $40.4(\mathrm{C})$ \\
\hline \multicolumn{5}{|l|}{ Unselected } \\
\hline Median OS (months) & - & - & NR (PC) vs. $11.3(\mathrm{C})$ & $15.9(\mathrm{PC})$ vs. $11.3(\mathrm{C})$ \\
\hline HR (95\% Cl) & - & - & $0.49(0.38-0.64)$ & $0.64(0.49-0.85)$ \\
\hline Median PFS (months) & - & - & $8.8(\mathrm{PC})$ vs. $4.9(\mathrm{C})$ & 6.4 (PC) vs. $4.8(\mathrm{C})$ \\
\hline $\mathrm{HR}(95 \% \mathrm{Cl})$ & - & - & $0.52(0.43-0.64)$ & $0.56(0.45-0.70)$ \\
\hline ORR (\%) & - & - & $47.6(\mathrm{PC})$ vs. $18.9(\mathrm{C})$ & 57.9 (C) vs. 38.4 (C) \\
\hline
\end{tabular}

*Updated survival analysis with extended follow-up. TPS, tumor proportion score; P, Pembrolizumab; C, Chemotherapy; PC, Pembrolizumab + Chemotherapy; NR, not reached; OS, overall survival; PFS, progression-free survival; ORR, objective response rate; HR, hazard ratio; CI, confidence interval; -, not reported; NSCLC, non-small-cell lung cancer. 
disease in whom sequential therapies may not be feasible. In the latter circumstance, we would favor combination chemoimmunotherapy regardless of tumor PD-L1 TPS.

\section{Cases from the clinic: ICls in real-world settings}

Tumor PD-L1 TPS $\geq 50 \%$ without actionable genomic alterations

A 63 -year-old man with a 40 pack-year tobacco history presents with advanced lung adenocarcinoma. Initial clinical evaluation shows no brain metastases and minimal diseaserelated symptoms. ECOG PS is 0. Tumor molecular profiling demonstrates a KRAS G12C mutation and no other actionable genomic alterations; tumor PD-L1 TPS is $90 \%$.

Recommended palliative therapy: on the basis of the KEYNOTE-024 study, single agent pembrolizumab given intravenously (IV) every 3 weeks for up to 2 years was recommended.

Clinical outcome: the patient has experienced a sustained partial response for $>18$ months and with plans for continued therapy to complete 2 years.

\section{Tumor PD-L1 TPS 0\% without actionable genomic alterations}

A 62 -year-old man with a 45 pack-year tobacco history presents with advanced lung adenocarcinoma, hypertrophic osteoarthropathy, cardio-pulmonary symptoms, and asymptomatic brain metastases. ECOG PS is 1. Tumor molecular profiling shows a KRAS G12D mutation and no other actionable genomic alterations; tumor PD-L1 TPS is $0 \%$.

Recommended palliative therapy: on the basis of KEYNOTE-189, the patient was commenced on combination therapy with carboplatin, pemetrexed, and pembrolizumab IV every 3 weeks.

Clinical outcome: The patient received two cycles of therapy with symptomatic improvement, but radiographically stable disease. He has experienced limited toxicity during treatment thus far. He has been advised to continue combination therapy for a total of four cycles, then to transition to pemetrexed/pembrolizumab maintenance therapy IV every 3 weeks for up to 2 years barring disease progression/toxicity.
Tumor PD-L1 TPS <50\% with borderline PS without actionable genomic alterations

A 63-year-old woman with a 60 pack-year tobacco history presents with advanced lung adenocarcinoma and significant neurologic symptoms due to brain metastases. Functional status following brain-directed therapy is modest (ECOG PS 2). Tumor molecular profiling demonstrates no actionable genomic alterations, TMB of 12 mutations/mega base pair, and PD-L1 TPS 4\%. The patient has expressed a desire not to receive cytotoxic chemotherapy.

Recommended palliative therapy: extrapolating from the data presented in KEYNOTE-042, single agent pembrolizumab IV every 3 weeks for up to 2 years was recommended.

Clinical outcome: at 12 months, the patient has had a sustained partial response to therapy accompanied by improvement in PS and no significant treatment-related AEs. She will continue with the current therapy for up to 2 years, barring disease progression/toxicity.

\section{Tumor PD-L1TPS $\geq 50 \%$ in a patient with significant symptomatic disease burden without actionable genomic alterations}

A 70-year-old woman with a prior 10 pack-year tobacco history presents with advanced lung adenocarcinoma complicated by malignant pericardial and pleural effusions, multiple painful osseous metastases, and moderate cardiopulmonary symptoms. ECOG PS is 1 . Tumor molecular profiling shows a KRAS G12C mutation and no other actionable genomic alterations; tumor PD-L1 TPS is $90 \%$.

Recommended palliative therapy: on the basis of KEYNOTE-189 and due to the need for augmented initial response to therapy for clinically meaningful cytoreductive palliation, the patient was advised to proceed with combination carboplatin, pemetrexed, and pembrolizumab IV every 3 weeks.

Clinical outcome: the patient received chemoimmunotherapy for four cycles with clinical and radiographic response, followed by maintenance therapy with pemetrexed and pembrolizumab. Pembrolizumab was subsequently discontinued due to immune-related pneumonitis and nephritis. After a protracted period of disease control in excess of 1 year, disease progression on pemetrexed maintenance was observed. There was no response with re- 
exposure to ICI, and the patient subsequently died.

\section{Conclusions}

As seen in the landmark KEYNOTE trials and in real-world clinical scenarios, ICIs have ushered in an era in lung cancer care where simultaneously efficacious, durable, and tolerable therapies are at last available for an increasing number of our patients. KEYNOTE-024 and -042 have further augmented the therapeutic armamentarium by offering a single agent approach that can afford sustained palliation of disease and improved survival with limited toxicities. However, singular reliance on PD-L1 TPS as a biomarker for optimal patient and therapeutic selection may yet be too simplistic of an approach-and efforts are necessarily ongoing to further evolve a biomarker strategy that more fully captures the depth and complexity of clinical outcomes with ICIs in advanced NSCLC. Additionally, continuing to explore combination and sequential therapeutic strategies involving ICIs along with other immunomodulatory agents, chemotherapy, biologic agents, and local therapies remains a persistent mandate so that we may maximize the benefits of these agents across the spectrum of patients with advanced NSCLC. Much work remains to be done, but the progress made within the past decade surely serves as a reminder of how far we have come- and the distance yet to be traveled in the effort to offer each patient the most optimally durable, efficacious, and tolerable strategy for their care.

\section{Acknowledgments}

Funding: DB Costa was supported in part by funds from National Institutes of Health grant R37 CA218707.

\section{Footnote}

Conflicts of Interest: D Rangachari reports non-financial support (institutional research support) from BristolMyers Squibb, Novocure, and Abbvie/Stemcentrx; all outside the submitted work. DB Costa reports personal fees (consulting fees and honoraria) and non-financial support (institutional research support) from Takeda/Millennium Pharmaceuticals, personal fees (consulting fees) and nonfinancial support (institutional research support) from AstraZeneca, personal fees (honoraria) and non-financial support (institutional research support) from Pfizer, nonfinancial support (institutional research support) from
Merck Sharp \& Dohme Corporation, non-financial support (institutional research support) from Merrimack Pharmaceuticals, non-financial support (institutional research support) from Bristol-Myers Squibb, non-financial support (institutional research support) from Clovis Oncology, non-financial support (institutional research support) from Tesaro; all outside the submitted work. The other authors have no conflicts of interest to declare.

\section{References}

1. Herbst RS, Morgensztern D, Boshoff C. The biology and management of non-small cell lung cancer. Nature 2018;553:446-54.

2. Lin JJ, Cardarella S, Lydon CA, et al. Five-Year Survival in EGFR-Mutant Metastatic Lung Adenocarcinoma Treated with EGFR-TKIs. J Thorac Oncol 2016;11:556-65.

3. Pacheco JM, Gao D, Smith D, et al. Natural History and Factors Associated with Overall Survival in Stage IV ALKRearranged Non-Small Cell Lung Cancer. J Thorac Oncol 2019;14:691-700.

4. Shaw AT, Riely GJ, Bang YJ, et al. Crizotinib in ROS1rearranged advanced non-small-cell lung cancer (NSCLC): updated results, including overall survival, from PROFILE 1001. Ann Oncol 2019. [Epub ahead of print].

5. Gettinger S, Horn L, Jackman D, et al. Five-Year FollowUp of Nivolumab in Previously Treated Advanced NonSmall-Cell Lung Cancer: Results From the CA209-003 Study. J Clin Oncol 2018;36:1675-84.

6. Lindeman NI, Cagle PT, Aisner DL, et al. Updated Molecular Testing Guideline for the Selection of Lung Cancer Patients for Treatment With Targeted Tyrosine Kinase Inhibitors: Guideline From the College of American Pathologists, the International Association for the Study of Lung Cancer, and the Association for Molecular Pathology. J Thorac Oncol 2018;13:323-58.

7. Kalemkerian GP, Narula N, Kennedy EB, et al. Molecular Testing Guideline for the Selection of Patients With Lung Cancer for Treatment With Targeted Tyrosine Kinase Inhibitors: American Society of Clinical Oncology Endorsement of the College of American Pathologists/ International Association for the Study of Lung Cancer/ Association for Molecular Pathology Clinical Practice Guideline Update. J Clin Oncol 2018;36:911-9.

8. Doroshow DB, Sanmamed MF, Hastings K, et al. Immunotherapy in Non-Small Cell Lung Cancer: Facts and Hopes. Clin Cancer Res 2019. 
9. Reck M, Rodriguez-Abreu D, Robinson AG, et al. Updated Analysis of KEYNOTE-024: Pembrolizumab Versus Platinum-Based Chemotherapy for Advanced NonSmall-Cell Lung Cancer With PD-L1 Tumor Proportion Score of 50\% or Greater. J Clin Oncol 2019;37:537-46.

10. Gandhi L, Rodriguez-Abreu D, Gadgeel S, et al. Pembrolizumab plus Chemotherapy in Metastatic NonSmall-Cell Lung Cancer. N Engl J Med 2018;378:2078-92.

11. Borghaei H, Paz-Ares L, Horn L, et al. Nivolumab versus Docetaxel in Advanced Nonsquamous Non-Small-Cell Lung Cancer. N Engl J Med 2015;373:1627-39.

12. Brahmer J, Reckamp KL, Baas P, et al. Nivolumab versus Docetaxel in Advanced Squamous-Cell Non-Small-Cell Lung Cancer. N Engl J Med 2015;373:123-35.

13. Rittmeyer A, Barlesi F, Waterkamp D, et al. Atezolizumab versus docetaxel in patients with previously treated non-small-cell lung cancer (OAK): a phase 3, openlabel, multicentre randomised controlled trial. Lancet 2017;389:255-65.

14. Reck M, Rodriguez-Abreu D, Robinson AG, et al. Pembrolizumab versus Chemotherapy for PD-L1Positive Non-Small-Cell Lung Cancer. N Engl J Med 2016;375:1823-33.

15. Mok TSK, Wu YL, Kudaba I, et al. Pembrolizumab versus chemotherapy for previously untreated, PD-L1expressing, locally advanced or metastatic non-small-cell lung cancer (KEYNOTE-042): a randomised, open-label, controlled, phase 3 trial. Lancet 2019;393:1819-30.

16. Paz-Ares L, Luft A, Vicente D, et al. Pembrolizumab plus Chemotherapy for Squamous Non-Small-Cell Lung Cancer. N Engl J Med 2018;379:2040-51.

17. Johnson DB, Sullivan RJ, Menzies AM. Immune checkpoint inhibitors in challenging populations. Cancer 2017;123:1904-11.

18. Carmichael JA, Wing-San Mak D, O'Brien M. A Review of Recent Advances in the Treatment of Elderly and Poor Performance NSCLC. Cancers (Basel) 2018. doi: 10.3390/ cancers 10070236.

19. Cook MR, Kim C. Safety and Efficacy of Immune Checkpoint Inhibitor Therapy in Patients With HIV Infection and Advanced-Stage Cancer: A Systematic Review. JAMA Oncol 2019. [Epub ahead of print].

20. Ostios-Garcia L, Faig J, Leonardi GC, et al. Safety and Efficacy of PD-1 Inhibitors Among HIV-Positive Patients With Non-Small Cell Lung Cancer. J Thorac Oncol 2018;13:1037-42.
21. Leonardi GC, Gainor JF, Altan M, et al. Safety of Programmed Death-1 Pathway Inhibitors Among Patients With Non-Small-Cell Lung Cancer and Preexisting Autoimmune Disorders. J Clin Oncol 2018;36:1905-12.

22. Goldberg SB, Gettinger SN, Mahajan A, et al. Pembrolizumab for patients with melanoma or non-smallcell lung cancer and untreated brain metastases: early analysis of a non-randomised, open-label, phase 2 trial. Lancet Oncol 2016;17:976-83.

23. Havel JJ, Chowell D, Chan TA. The evolving landscape of biomarkers for checkpoint inhibitor immunotherapy. Nat Rev Cancer 2019;19:133-50.

24. Gainor JF, Shaw AT, Sequist LV, et al. EGFR Mutations and ALK Rearrangements Are Associated with Low Response Rates to PD-1 Pathway Blockade in Non-Small Cell Lung Cancer: A Retrospective Analysis. Clin Cancer Res 2016;22:4585-93.

25. Moya-Horno I, Viteri S, Karachaliou N, et al. Combination of immunotherapy with targeted therapies in advanced non-small cell lung cancer (NSCLC). Ther Adv Med Oncol 2018;10:1758834017745012.

26. Lisberg A, Cummings A, Goldman JW, et al. A Phase II Study of Pembrolizumab in EGFR-Mutant, PD-L1+, Tyrosine Kinase Inhibitor Naive Patients With Advanced NSCLC. J Thorac Oncol 2018;13:1138-45.

27. Negrao MV, Lam VK, Reuben A, et al. PD-L1 Expression, Tumor Mutational Burden, and Cancer Gene Mutations Are Stronger Predictors of Benefit from Immune Checkpoint Blockade than HLA Class I Genotype in Non-Small Cell Lung Cancer. J Thorac Oncol 2019. [Epub ahead of print].

28. Schoenfeld AJ, Arbour KC, Rizvi H, et al. Severe immune related adverse events are common with sequential PD(L)1 blockade and osimertinib. Ann Oncol 2019.

29. Lin JJ, Chin E, Yeap BY, et al. Increased Hepatotoxicity Associated with Sequential Immune Checkpoint Inhibitor and Crizotinib Therapy in Patients with Non-Small Cell Lung Cancer. J Thorac Oncol 2019;14:135-40.

Cite this article as: Piper AJ, Sehgal K, Costa DB, Rangachari D. Can PD-L1 tumor proportion score be used as the key to unlocking the KEYNOTE studies of pembrolizumab in advanced lung cancer? Transl Lung Cancer Res 2019;8(5):715722. doi: 10.21037/tlcr.2019.05.12 